\title{
Lower Limb Biomechanics and Muscle Function
}

Introduction While the basic principles of lower limb biomechanics have been explored extensively with respect to understanding performance, injury or disease, there is still a significant amount that we do not know, particularly at the level of muscle function and control. Biomechanics research has typically focused on performance outcomes that describe whole body or joint movement and inferred muscle function from these measurements. However, recent developments in measurement technologies have made it possible to zoom in and directly analyse individual muscle function. These approaches have provided a new understanding of how humans control movements, ranging from walking and running, to jumping and sprinting. This symposium will explore various concepts of lower limb biomechanics at different levels of organisation and under different locomotion conditions. The aim of the symposium is to: a) provide information about how specific lower limb muscles are used to control movement under different locomotion conditions; b) explore new methods that can provide detailed information about the control of movement at the level of individual muscles; $c$ ) develop a detailed understanding of how different aspects of muscle design can be used to understand how the body performs movements and the constraints this imposes on our performance.

Main body The session will start with a brief overview of how new research methodologies can be used to gain new insight into muscle function. Four research papers will follow this, where a variety of innovative biomechanical technologies have been used to provide new insight into muscle function of the lower limb. Paper 1 will explore muscle function at the limits of human performance and examine lower limb biomechanics during sprinting. Paper 2 will then provide new insights into the role of the foot musculature in controlling foot stiffness during running. Paper 3 will investigate the role that elastic structures in muscles play in absorbing energy at foot contact during walking. Paper 4 will then explore how using external devices that are aimed to assist humans (e.g. exoskeletons) may have unexpected consequences in terms of muscle function. Following the four papers, a discussion about how these findings might be important for understanding performance, injury or disease will be led by the chair.

Introduction: Innovative methodologies for understanding muscle function in the lower limb (Dr Glen Lichtwark)

Paper 1: Lower limb muscular strategies for increasing running speed (Dr Nick Brown)

Paper 2: Active regulation of foot stiffness during running (Mr Luke Kelly)

Paper 3: The role of the tibialis anterior muscle and tendon in absorbing energy during walking ( $\mathrm{Dr}$ Glen Lichtwark) Paper 4: Does unloading muscles save you energy? - Paradoxical effects of spring-loaded ankle exoskeletons on plantar flexor muscle mechanics and energetics (Dr Dominic Farris) 